\title{
Preliminary results of simultaneous integrated boost intensity-modulated radiation therapy based neoadjuvant chemoradiotherapy on locally advanced rectal cancer with clinically suspected positive lateral pelvic lymph nodes
}

\author{
Jian-Hao Geng ${ }^{1 \#}$, Yang-Zi Zhang ${ }^{1 \#}$, Yong-Heng Li $^{1 \#}$, Shuai Li $^{1}$, Lin Wang ${ }^{2}$, Zhi-Long Wang ${ }^{3}$, \\ Xiang-Gao Zhu ${ }^{2}$, Zhao-De $\mathrm{Bu}^{2}$, Zi-Yu Li ${ }^{2}$, Xiang-Qian Su${ }^{2}$, Yong Cai ${ }^{1}$, Ai-Wen Wu ${ }^{2}$, Wei-Hu Wang ${ }^{1}$ \\ ${ }^{1}$ Key Laboratory of Carcinogenesis and Translational Research (Ministry of Education/Beijing), Department of Radiation Oncology, Peking \\ University Cancer Hospital and Institute, Beijing, China; ${ }^{2}$ Key Laboratory of Carcinogenesis and Translational Research (Ministry of Education/ \\ Beijing), Department of Gastrointestinal Surgery, Peking University Cancer Hospital and Institute, Beijing, China; Key Laboratory of \\ Carcinogenesis and Translational Research (Ministry of Education/Beijing), Department of Radiology, Peking University Cancer Hospital and \\ Institute, Beijing, China \\ Contributions: (I) Conception and design: WH Wang; (II) Administrative support: WH Wang, AW Wu; (III) Provision of study materials or patients: \\ All authors; (IV) Collection and assembly of data: JH Geng, YZ Zhang, YH Li; (V) Data analysis and interpretation: S Li; (VI) Manuscript writing: \\ All authors; (VII) Final approval of manuscript: All authors. \\ \#These authors contributed equally to this work. \\ Correspondence to: Prof. Wei-Hu Wang, MD; Prof. Ai-Wen Wu, MD. 52 Fucheng Rd, Haidian District, Beijing 100142, China. \\ Email: wangweihu88@163.com; wuaw@sina.com.
}

Background: Lateral pelvic lymph node (LPLN) is approximately $11-14 \%$ and always associated with poorer prognosis. This study investigated the efficacy and safety of simultaneous integrated boost intensitymodulated radiation therapy (SIB-IMRT) based on neoadjuvant chemoradiotherapy (NCRT) on locally advanced rectal cancer (LARC) patients with clinically suspected positive LPLNs.

Methods: We retrospectively screened distal LARC patients with NCRT in our center from May 2016 and June 2019. The diagnostic criteria of positive LPLN were nodes of over $7 \mathrm{~mm}$ in short axis and irregular border or mixed-signal intensity. All patients with clinically suspected positive LPLN received 56-60 Gy SIB-IMRT in the LPLN area. Concurrent chemotherapy regimens were capecitabine as monotherapy treatment or in combination with oxaliplatin. The toxicities, local-regional recurrence (LRR), and diseasefree survival (DFS) were investigated.

Results: Fifty-two eligible patients with clinically suspected positive LPLN were screened and analyzed. The median distance from the distal tumor to the anal verge was $4 \mathrm{~cm}$ (range, $0-8 \mathrm{~cm}$ ), while magnetic resonance imaging (MRI) analysis revealed the median short diameter of the pelvic LPLN to be $8 \mathrm{~mm}$ (range, 7-20 mm). There were 28 (53.8\%) mesorectal fascia (MRF) positive and 22 (42.3\%) extramural venous invasion (EMVI) positive patients. A radiotherapy dose of 41.8 Gy was administered to the pelvic area, while the LPLN received a median SIB dose of 60.0 Gy (range, 56-60 Gy) across 22 fractions. Synchronous capecitabine with or without oxaliplatin was administered during radiotherapy. In summary, $15(28.8 \%)$ patients displayed grade 2-3 radiation-related toxicity, 8 (15.4\%) patients underwent additional LPLN dissection, and positive nodes (26 nodes in total) were not observed. One patient suffered a LLR in the presacral region. The median follow-up duration was 21.2 months (range, 4.7-45.0 months), while the duration of 1 - and 2-year DFS were $89.9 \%$ and $74.6 \%$, respectively. Patients did not display LPLN recurrence.

Conclusions: The safety and efficacy of SIB-IMRT on clinically suspected positive LPLN of LARC patients were deemed acceptable. Patients did not exhibit in-field LPLN recurrence after NCRT combined with single total mesorectal excision (TME). 
Keywords: Locally advanced rectal cancer (LARC); lateral pelvic lymph nodes (LPLNs); simultaneous integrated boost; chemoradiotherapy; disease free survival (DFS)

Submitted May 20, 2020. Accepted for publication Oct 23, 2020.

doi: $10.21037 /$ atm-20-4040

View this article at: http://dx.doi.org/10.21037/atm-20-4040

\section{Introduction}

Colorectal cancer is the fifth most common form of malignant tumors in China, with rectal cancer accounting for over $50 \%$ of the entire patient population $(1,2)$. Most rectal patients were at locally advanced stages $(\mathrm{cT} 3-4 / \mathrm{N}+)$ at the time of diagnosis, due to the mild nature of occult symptoms. Treatment failure in these patients is primarily attributed to local-regional recurrence (LRR). Neoadjuvant chemoradiotherapy (NCRT) has become the standard treatment for locally advanced rectal cancer (LARC) during the past decade (3-8). It can improve local-regional control and didn't increase surgery complications rate when compared with adjuvant chemoradiotherapy or neoadjuvant radiotherapy. NCRT results in clinically significant tumor regression, where a pathologic complete response (pCR) was a reliable indicator of perfect disease-free survival (DFS) $(3,4)$.

Total mesorectal excision (TME) improved the surgical management of rectal cancer and substantially reduced the local recurrence rate (5-7). However, TME only removes the primary lesion with the mesorectum, where the suspected positive LPLN cannot be eliminated. Distal rectal cancer has an added consideration of poorer prognosis and a positive rate of lateral pelvic lymph node (LPLN) of approximately 11-14\% (8-10). In Japan, TME combined with lateral pelvic lymph node dissection (LPLD) was recommended for rectal cancer that extended below the peritoneal reflection due to potential metastases (11). Nevertheless, LPLD need longer operation duration, increased intraoperative bleeding, and has a more significant impact on the pelvic autonomic nerve then resulting in dysuria to a certain extent and sexual dysfunction $(12,13)$. Oncologists in western countries view NCRT as a viable means of treating relatively small LPLN and achieves similar LLR with LPLD (14). Nevertheless, for the larger LPLN, standard chemoradiotherapy may not be sufficient to gain local control in the lateral pelvic region (15-17).

Simultaneous integrated boost intensity-modulated radiation therapy (SIB-IMRT) provides a strong rationale for intensified radiotherapy, delivering higher doses in a shorter timeframe. Studies that evaluated the role of intensified radiation dose with 5FU-based chemotherapy on primary tumors of LARC found a correlation between increased dose and higher pCR rates, along with acceptable toxicity (18). Whether radiation dose escalation is effective for clinically suspected positive LPLN is still unclear. Therefore, this study evaluated the efficacy and safety of SIB-IMRT based NCRT on LARC patients with clinically diagnosed metastatic LPLNs. We present the following article following the STROBE reporting checklist (available at http://dx.doi.org/10.21037/atm-20-4040).

\section{Methods}

From May 2016 and June 2019, we retrospectively screened the data of distal LARC patients who received NCRT in our center. The inclusion criteria were as follows: (I) the tumor located within $8 \mathrm{~cm}$ from the anal verge and histologically confirmed cancer; (II) clinical staging T3-4 or positive lymph nodes, without any distant metastases; (III) clinically suspected positive lymph nodes in internal iliac or obturator region; (IV) aged over 18 years; (V) Eastern Cooperative Oncology Group (ECOG) performance status of $0-1$ and without any severe complications.

Every patient signed an informed consent form before chemoradiotherapy. The following assessment results were retrospectively recorded: clinical history, physical examinations, colonoscopy, pathologic and radiology examinations, blood tests. The study was conducted in accordance with the Declaration of Helsinki (as revised in 2013). The study was approved by the committee board of Beijing cancer Hospital (No.: 2020YJZ68).

\section{Pelvic magnetic resonance imaging (MRI) assessment}

All patients underwent pelvic MRI (1.5T or $3.0 \mathrm{~T}$ ) examination before NCRT. At least 3 T2-weighted section sequences were used: sagittal, coronal, and oblique axial planes. MRI images were evaluated by a radiologist with 10 years of experience in abdominal pelvic radiology, and 

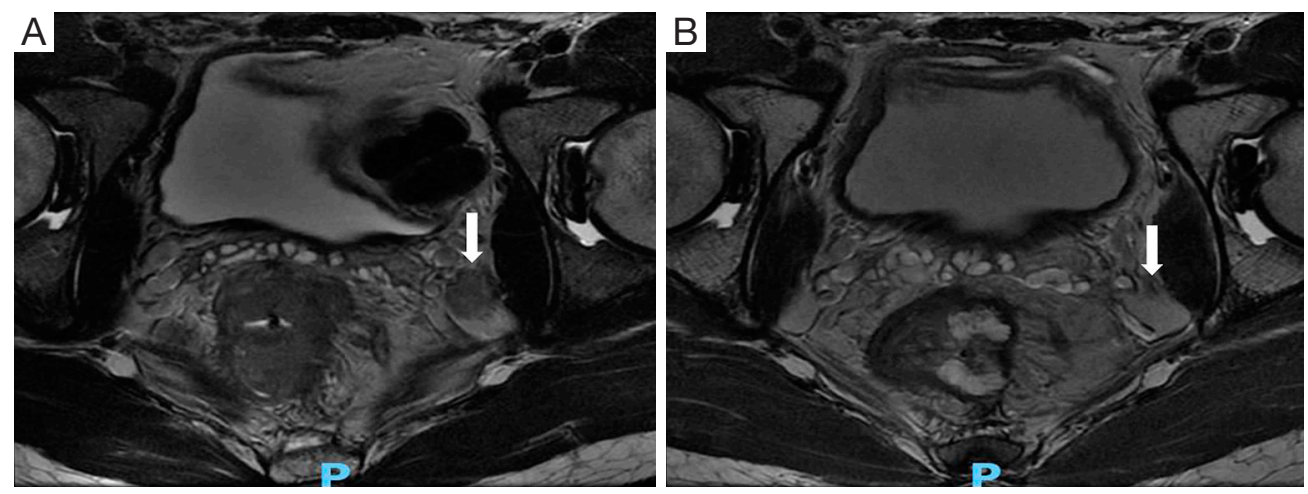

Figure 1 Comparison of MRI image of one patient with clinically positive LPLD before and after NCRT. (A) One patient's primary MRI, the white arrow indicated the clinically positive LPLN with a short axis of $14 \mathrm{~mm}$; (B) preoperative MRI showed the previous LPLN nearly disappeared after NCRT and the LPLD was exempted. MRI, magnetic resonance imaging; LPLD, lateral pelvic lymph node dissection; NCRT, neoadjuvant chemoradiotherapy; LPLN, lateral pelvic lymph node.

a radiation oncologist specifically with 5 years' experience in rectal radiotherapy. The short-axis diameter of LPLNs was measured by the radiation oncologist and screened for highly suspicious lymph nodes. The radiologist subsequently assessed the border contour and presence of mixed-signal intensity of the suspected LPLNs. The diagnostic criteria of clinically suspected positive LPLN were: short diameter $\geq 7 \mathrm{~mm}$, with irregular lymph node edge or mixed signals $(19,20)$. The mesorectal fascia (MRF) and extramural vascular invasion (EMVI) were also evaluated.

After NCRT, all patients underwent MRI re-evaluation to determine the next course of appropriate treatment, where the majority of patients were advised to attend the multidisciplinary team (MDT) consultation. As metastatic lateral lymph nodes will shrink after radiotherapy, their edge and signal are not viable references for negative diagnoses. The short diameter of the lateral lymph node $<5 \mathrm{~mm}$ was considered as imaging negative. The radiation oncologist and radiologist read the MRI scans together to assess the volume change of LPLN (Figure 1).

\section{Neoadjuvant chemoradiotherapy}

All patients underwent contrast-enhanced computed tomography (CT) and MRI simulations with $5 \mathrm{~mm}$ slice thickness, in the supine position with a full bladder and empty rectum. Target delineation was based on CT and MRI fused imaging. The gross tumor volume (GTV) consisted of GTVp and GTVn. GTVp was defined as the primary lesion and the metastatic mesorectal lymph nodes.
GTVn was defined as metastatic LPLNs. Clinical tumor volume (CTV) was defined as the mesorectal and presacral regions, obturator, and internal iliac lymph node drainage area, with a $2-\mathrm{cm}$ margin from the superior and inferior extents of the primary lesion in the rectum. Planning gross target volume (PGTVp/PGTVn) and planning target volume (PTV) was determined by the addition of a $0.5-\mathrm{cm}$ margin to the GTVp/GTVn and CTV, respectively.

All patients underwent SIB-IMRT. PGTVp was prescribed 2.3 Gy per fraction, totaling 50.6 Gy in 22 fractions. PTV was prescribed at 22 fractions at $1.9 \mathrm{~Gy}$ per fraction, while PGTVn was prescribed 2.5-2.7 Gy per fraction, totaling 56-60 Gy in 22 fractions [2 Gy equivalent dose $(\mathrm{EQD} 2)=59-65.3 \mathrm{~Gy}$, the $\alpha / \beta$ of rectal cancer is $5.08(21)$ ]. The details of one patient are illustrated in Figure 2.

Patients exhibiting risk factors such as positive MRF or EMVI were recommended weekly concurrent chemotherapy of CapeOX (oxaliplatin $50 \mathrm{mg} / \mathrm{m}^{2}$ intravenous infusion once a week), and capecitabine $\left(825 \mathrm{mg} / \mathrm{m}^{2}\right.$ orally twice daily, 5 days/week), while others received capecitabine $\left(825 \mathrm{mg} / \mathrm{m}^{2}\right.$ orally twice daily, 5 days/week) during radiotherapy (22).

Organs at risk (OARs) were identified as the bladder, sigmoid, small bowel, and femoral heads. The recommended dose constraints referred to the QUANTEC review (23).

\section{Surgical treatment and pathology procedures}

Patients received surgery approximately 8-12 weeks after completion of NCRT. Surgical techniques included laparoscopic-assisted resection and open resection. 

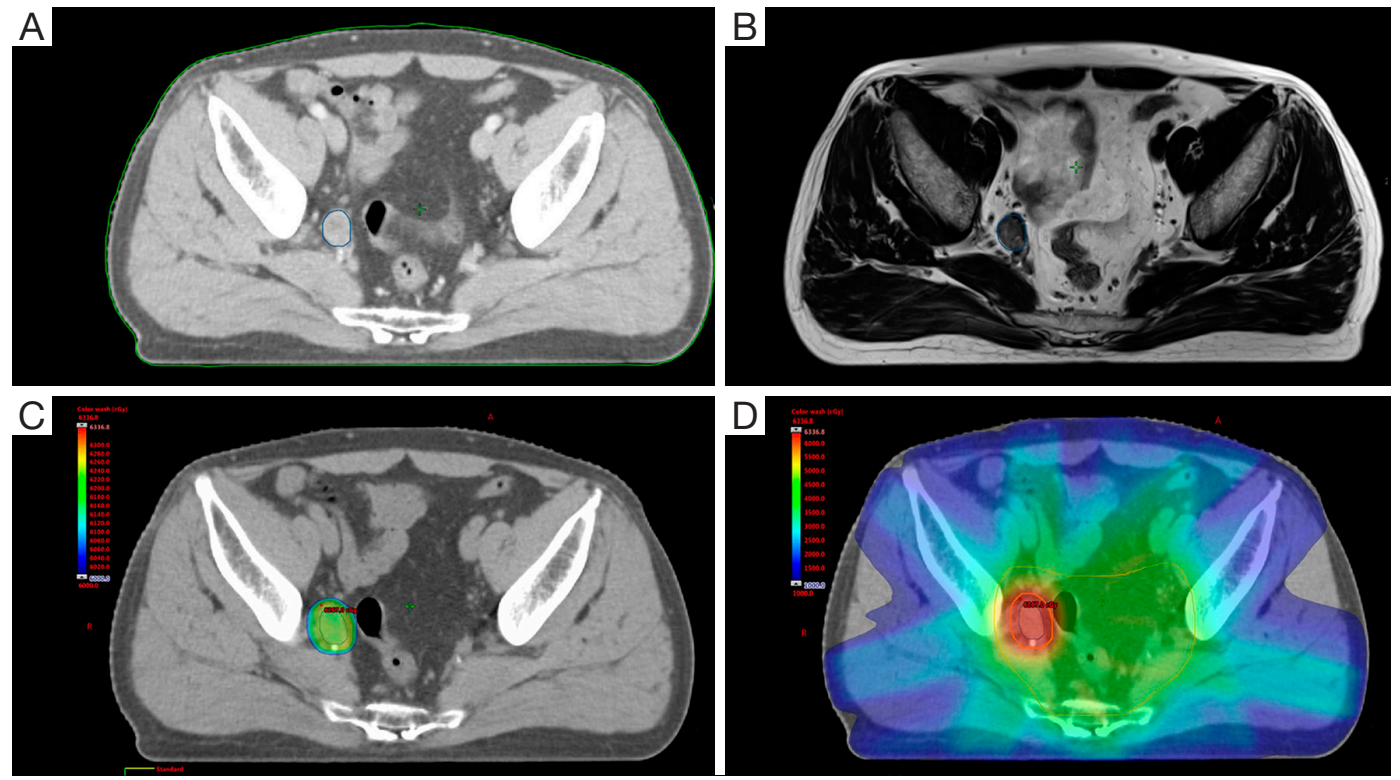

Figure 2 A case of rectal cancer with lateral pelvic lymph node (LPLN) metastasis. (A) Image of CT simulation, bule circle indicates the clinically positive LPLN with short axis of $12 \mathrm{~mm}$; (B) image of T2-weighted section sequences of the MRI simulation, blue lines indicates the clinically positive LPLN; (C) dose-color wash of the PGTVn; (D) dose-color wash of the PTV (orange lines) and PGTVn (bold orange lines). CT, computed tomography; MRI, magnetic resonance imaging; PGTV, planning gross target volume; PTV, planning target volume.

The patterns included low anterior resection (LAR), abdominoperineal resection (APR), local excision, and Hartmann surgery. However, LPLD was undertaken, based on MDT consultation. TME \pm LPLD was performed with the same standard technique among all the patients.

Specimens from each patient underwent a standardized pathology examination. Tumors were staged according to the seventh edition of the American Joint Committee on Cancer TNM classification.

\section{Neoadjuvant/adjuvant chemotherapy}

Neoadjuvant chemotherapy was administered based on the doctors' discretion and patients' consent. Principle drug regimens were capecitabine with or without oxaliplatin. Adjuvant chemotherapy was recommended for all patients exhibiting risk factors post-surgery. Chemotherapy regimens include CapeOX, FOLFOX4, and mFOLFOX6 regime based on Chinese Society of Clinical Oncology (CSCO) guidelines.

\section{Safety evaluation and follow-up}

Adverse event assessments were performed for all patients every week during the NCRT and subsequently monthly after the treatment. Treatment-related toxicities were assessed according to the Common Terminology Criteria Adverse Events Version 4.0 (CTCAE v4.0).

Patients were followed up every 3 months during the first 2 years post-surgery, every 6 months for the next 3 years, and then annually for the subsequent 5 years. Evaluations consisted of physical examinations, chest CT, pelvic CT or MRI, carcinoembryonic antigen (CEA) and other blood assessments. Colonoscopy was performed if clinically required.

\section{Study endpoints and statistical analysis}

The LRR was identified as the primary endpoint. It was defined as the time from the first NCRT administration to the date of LRR. The secondary endpoints were identified as toxicities and DFS. DFS was measured from the first NCRT administration to the date of any type of recurrence or death from any cause.

Statistical Package analyzed data for the Social Sciences (IBM Corp., SPSS Statistics for Windows, v. 22.0). Categorical variables were compared using $\chi^{2}$ tests or the Fisher exact test. The rates of LRR and DFS were 
Table 1 Baseline characteristics of 52 patients

\begin{tabular}{|c|c|}
\hline Characteristic & N (\%) \\
\hline Age [range] (years) & 56 [30-74] \\
\hline \multicolumn{2}{|l|}{ Gender, n (\%) } \\
\hline Male & $36(69.2)$ \\
\hline Female & $16(30.8)$ \\
\hline \multicolumn{2}{|l|}{ ECOG, n (\%) } \\
\hline 0 & 27 (51.9) \\
\hline 1 & $25(48.1)$ \\
\hline \multicolumn{2}{|l|}{ Pretreatment CEA, n (\%) } \\
\hline$\geq 5 \mathrm{ng} / \mathrm{mL}$ & $28(53.8)$ \\
\hline$<5 \mathrm{ng} / \mathrm{mL}$ & $20(38.5)$ \\
\hline Unidentified & $4(7.7)$ \\
\hline \multicolumn{2}{|l|}{ Clinical T stage, n (\%) } \\
\hline T3 & $44(84.6)$ \\
\hline $\mathrm{T} 4$ & $8(15.4)$ \\
\hline \multicolumn{2}{|l|}{ Clinical N stage, n (\%) } \\
\hline $\mathrm{N} 1$ & $13(25.0)$ \\
\hline N2 & $39(75.0)$ \\
\hline Distance from anal [range] (cm) & $4[0-8]$ \\
\hline Short diameter of LPLN [range] (mm) & 8 [7-20] \\
\hline \multicolumn{2}{|l|}{ MRF status, n (\%) } \\
\hline Positive & $28(53.8)$ \\
\hline Negative & $24(46.2)$ \\
\hline \multicolumn{2}{|l|}{ EMVI status, n (\%) } \\
\hline Positive & $22(42.3)$ \\
\hline Negative & $30(57.7)$ \\
\hline \multicolumn{2}{|l|}{ Prescribe dose of LPLN, n (\%) } \\
\hline$<60$ Gy & $21(40.4)$ \\
\hline 60 Gy & $31(59.6)$ \\
\hline \multicolumn{2}{|l|}{ Synchronous chemotherapy, n (\%) } \\
\hline Capecitabine & $32(61.5)$ \\
\hline CapOX & $20(38.5)$ \\
\hline \multicolumn{2}{|l|}{ Neoadjuvant CT, n (\%) } \\
\hline Yes & $21(40.4)$ \\
\hline No & $31(59.6)$ \\
\hline
\end{tabular}

ECOG, Eastern Cooperative Oncology Group; CEA, carcino-embryonic antigen; MRF, mesorectal fasciae; EMVI, external vascular invasion; LPLN, lateral pelvic lymph node; CapOX, capecitabine and oxaliplatin. calculated with the Kaplan-Meier method. $\mathrm{P}<0.05$ was considered statistically significant.

\section{Results}

\section{Patients characteristics}

A total of 586 LARC who receive NCRT were screened in our center, and 52 patients with positive clinical LPLN who received neoadjuvant SIB-IMRT, based chemoradiotherapy and operation, were included (Table 1). The median short diameter of lateral lymph nodes was $8 \mathrm{~mm}$ (range, 7-20 mm). Concerning clinical T/N staging diagnoses by MRI, 39 patients (75.0\%) were diagnosed as $\mathrm{cN} 2$, and $8(15.4 \%)$ patients were staged T4. There were $28(53.8 \%)$ MRF positive, and 22 (42.3\%) EMVI positive patients. During treatment, $31(59.6 \%)$ patients received $60 \mathrm{~Gy}$ to the lateral lymph node, and other patients were below this dose, 32 (61.5\%) patients were assigned to synchronous single capecitabine agent. Furthermore, $21(40.4 \%)$ patients received additional neoadjuvant chemotherapy.

\section{Surgery and pathology}

After rigorous radiological and physical examinations (range, 8-17 weeks after chemoradiotherapy), all patients underwent rectal tumor excisions in the surgical department of our center. Among them, 23 patients received APR, and 23 received LAR. Eight patients (15.4\%) underwent additional LPLD, and positive nodes (26 nodes in total) were not observed. The details between the two patient groups are displayed in Table 2.

The pathology of the total specimen indicated that the R0 resection was achieved in $51(98.1 \%)$ patients. Ten (19.2\%) patients achieved pCR, and 35 (67.3\%) patients achieved ypN0 staging. Pathology $\mathrm{T}$ and $\mathrm{N}$ downstaging were found in $34(65.4 \%)$ and $50(96.2 \%)$ patients, respectively.

\section{Treatment-related toxicity}

During NCRT, no patients underwent toxicity-related radiotherapy interruption or dose reduction. Also, no treatment-related deaths were observed. Fifty-one (98.1\%) patients completed full dose synchronous chemotherapy without any incidences of grade 4 toxicity. Fifteen (28.8\%) patients exhibited grade 2-3 radiation-related toxicity, with 
Table 2 Details of surgery and pathology results

\begin{tabular}{|c|c|c|c|}
\hline Variables & $\begin{array}{l}\text { Single surgery } \\
\qquad(\mathrm{N}=44)\end{array}$ & $\begin{array}{l}\text { Surgery with } \\
\text { LPLD }(\mathrm{N}=8)\end{array}$ & $P$ value \\
\hline $\begin{array}{l}\text { Reassessment for LPLN } \\
\text { after CRT (short axis) }\end{array}$ & & & 0.192 \\
\hline$\geq 4 \mathrm{~mm}$ & 37 & 7 & \\
\hline$<4 \mathrm{~mm}$ & 7 & 1 & \\
\hline Surgery method & & & 0.846 \\
\hline APR & 20 & 3 & \\
\hline LAR & 19 & 4 & \\
\hline Local excision & 2 & 0 & \\
\hline Hartmann & 3 & 1 & \\
\hline $\begin{array}{l}\text { Surgery time } \\
\text { [range] (min) }\end{array}$ & $\begin{array}{c}197 \\
{[60-410]}\end{array}$ & $\begin{array}{c}203 \\
{[63-360]}\end{array}$ & - \\
\hline $\begin{array}{l}\text { Amount of bleeding } \\
\text { [range] }(\mathrm{mL})\end{array}$ & $\begin{array}{c}100 \\
{[20-300]}\end{array}$ & $\begin{array}{c}100 \\
{[30-200]}\end{array}$ & - \\
\hline $\begin{array}{l}\text { Hospital stays } \\
\text { after surgery [range] }\end{array}$ & 7 [4-48] & 9 [6-14] & - \\
\hline pT Stage & & & 0.924 \\
\hline TO & 10 & 2 & \\
\hline $\mathrm{T} 1$ & 3 & 1 & \\
\hline $\mathrm{T} 2$ & 12 & 3 & \\
\hline T3 & 18 & 2 & \\
\hline $\mathrm{T} 4$ & 1 & 0 & \\
\hline pN stage & & & 0.783 \\
\hline No & 31 & 5 & \\
\hline N1 & 12 & 3 & \\
\hline N2 & 1 & 0 & \\
\hline $\mathrm{pCR}$ & 9 & 1 & 0.599 \\
\hline TRG grade & & & 0.375 \\
\hline 0 & 10 & 2 & \\
\hline 1 & 15 & 5 & \\
\hline 2 & 18 & 1 & \\
\hline 3 & 1 & 0 & \\
\hline
\end{tabular}

LPLD, lateral pelvic lymph node dissection; LPLN, lateral pelvic lymph node; CRT, chemoradiotherapy; APR, abdominoperineal resection; LAR, low anterior resection; pCR, pathology complete response; TRG, tumor regression grade.
Table 3 Details of treatment-related toxicities

\begin{tabular}{ll}
\hline Variables & $\mathrm{N}(\%)$ \\
\hline CRT-related toxicity (grade 2-3) & $7(13.5)$ \\
Leukopenia & $8(15.4)$ \\
Proctitis & $3(5.8)$ \\
Radiodermatitis & $1(1.9)$ \\
Anemia & $1(1.9)$ \\
Ventosity & \\
Postoperative complication & $3(5.8)$ \\
Small-bowel obstruction & $2(3.8)$ \\
Abdominal wound infections & $2(3.8)$ \\
Perineal wound infections & $1(1.9)$ \\
Anastomotic leakage & $1(1.9)$ \\
Urological &
\end{tabular}

$6(18.8 \%)$ observed in the capecitabine group and 9 (45.0\%) in the CapOX group, among whom 1 patient displayed grade 3 neutropenia. The incidence rates were significantly different between the two synchronous chemotherapy groups $(\mathrm{P}=0.042)$. The details of the toxicities are exhibited in Table 3.

While postoperative complications were relatively low (21.1\%), the most commonly observed were small-bowel obstruction (3 patients, $5.8 \%$ ) and infections in the perineal wound ( 2 patients, $3.8 \%$ ) or abdominal wound ( 2 patients, $3.8 \%)$. The incidence rate of complications was similar and independent of LPLD (25.0\% vs. $20.5 \%, \mathrm{P}=0.772)$.

\section{Long-term prognosis}

The median follow-up duration was 21.2 months (range, 4.7-45.0 months). One patient displayed LRR in the presacral region, and also exhibited inguinal lymph node metastasis. None patients displayed LRR in obturator or internal iliac lymph node drainage area, which received high-dose radiotherapy. Eleven patients developed distant metastases (3 with lung metastasis, 2 with brain metastasis). Three patients died of multi metastases, and another 
A

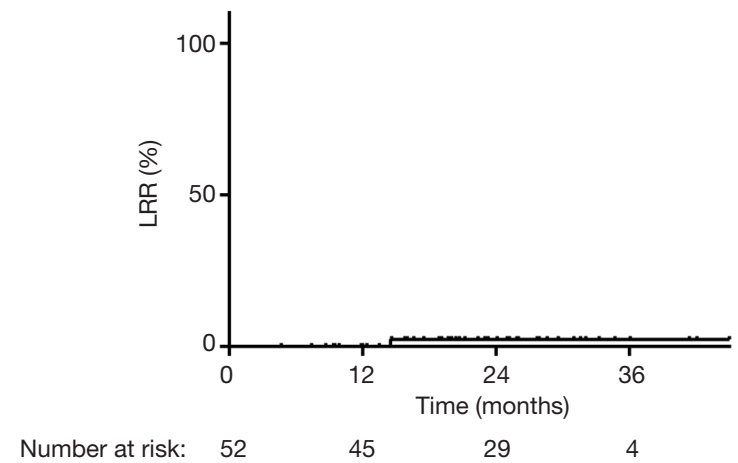

B

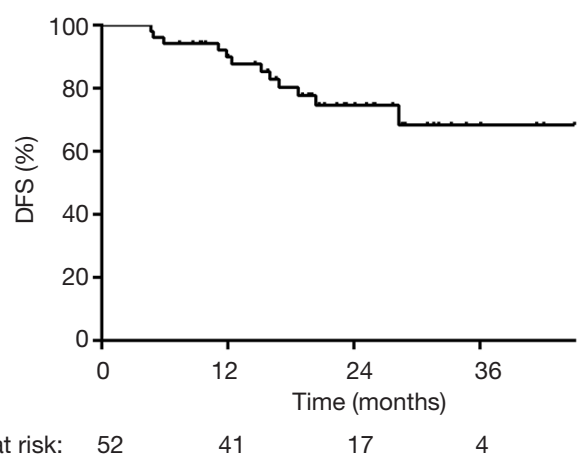

Figure 3 The Kaplan-Meier curve of local-regional recurrence (LRR) (A) and disease-free survival (DFS) (B).

expired from an abdominal infection. All deaths were observed during the follow-up period.

The 1 - and 2-year LRR were $0 \%$ and $2.4 \%$, respectively, for all patients. Meanwhile, no recurrences were observed in the initial LPLN region. One- and 2-year DFS was $89.9 \%$ and $74.6 \%$, respectively (Figure 3 ).

\section{Discussion}

In this retrospective study, only 8 patients were found to require LPLD after SIB-IMRT, with the remaining patients identified as negative for LPLN. During 21.2 months of follow-up, the 2-year LRR and DFS were $2.4 \%$ and $74.6 \%$, respectively. The preliminary results show that the toxicity was acceptable without LPLN recurrence in the SIB-IMRT region.

Since NCRT combined with TME has become the established treatment for distal LARC, the local recurrence rates within the anastomotic stoma and mesorectal area have been significantly reduced. Nevertheless, lateral pelvic recurrence has emerged as a dominant issue. One study from Korea indicated that lateral pelvic recurrence contributed to $80 \%$ of LRR for LARC after NCRT and surgery. Distal rectal cancers displayed a higher susceptibility to LPLN metastasis. This is due to the anatomical characteristics around lymph node drainage: lymphatic vessels below the peritoneal reflection converge into the internal iliac lymph nodes, which subsequently drain to other lateral lymph nodes (9).

However, the diagnostic criteria for positive LPLN are still controversial. Brown and his team used sectioned surgical specimens to evaluate the suspected lymph nodes. The results indicated irregular border or mixed signals as an indicator of high susceptibility toa positive lymph node (24). The JCOG0212 study analyzed 328 patients who received LPLD. The results demonstrated that 24 (7.3\%) patients were pathologically undergoing LPLN metastasis.

Further analysis suggested that a lower tumor location $(\mathrm{P}=0.025)$ and higher short-axis diameter $(\mathrm{P}=0.002)$ were linked to pathologically positive LPLN. Our study focused on patients with distal rectal cancers $(<8 \mathrm{~cm}$ from anal edge). The diagnostic criteria for positive LPLN in this study consisted of short-axis over $7 \mathrm{~mm}$ and irregular morphology of mixed signals. Our data demonstrated that the rate of positive LPLN in distal rectal cancer patients was $8.9 \%(52 / 586)$, similar to the result reported in the Japanese literature.

For patients with positive LPLN, additional LPLD may bring survival benefits. However, some studies suggested that the LPLD could affect men's sexual activity and urine function $(12,13)$. Therefore, non-invasive treatments which enhance the local treatment effect and ensure safety was urgently needed. With the development of precise radiotherapy technology, the SIB-IMRT technique can optimize the dose applied to the target area while protecting normal tissues. The higher dose has previously achieved satisfactory results in the treatment of primary rectal lesions. According to Burbach's meta-analysis of 14 studies, the ideal pCR rate and acceptable side effects were achieved at the radiation dose of $\geq 60$ Gy, in 1,106 LARC patients who underwent NCRT and 487 patients treated with radiation boost (25). Marco analyzed 76 LARC patients who received SIB-IMRT. The median SIB dose was identified as $54 \mathrm{~Gy}$, with $20(26.3 \%)$ patients shown to achieve pCR. However, the efficacy of radiation dose escalation for clinically suspected positive LPLN remains unclear. 
To our knowledge, this is the first retrospective study aimed to explore the potential of SIB-IMRT on clinically suspected positive LPLNs followed by TME in achieving relatively good prognoses without increasing side effects. In this study, patients with positive LPLN received 56-60 Gy by SIB-IMRT (EQD2 =59-65.3 Gy). Only 8 patients received LPLD after SIB-IMRT, and positive LPLNs were not detected. Only 1 patient displayed LRR. However, the area of recurrence was not the target site, which received a high-dose of radiotherapy. The LRR of the boost irradiation area was $100 \%$. No LPLN recurrence was noted in the SIB-IMRT region. The results illustrated good regulation of the clinically positive LPLN after SIB-IMRT.

Preliminary results demonstrated acceptable acute toxicity. In this study, grade 2-3 toxicities during SIB-IMRT were observed at only $28.1 \%$. Additionally, most of these toxicities were attributed to synchronous chemotherapy. Severe perioperative complications were not exhibited, and the rate of surgical complications was also deemed acceptable. However, long-term toxicities are still unclear and require further investigations.

In clinical practice settings, patients with clinically suspected positive LPLN consistently exhibit additional high-risk factors such as positive MRF and EMVI status, which are also linked with poor local control and survival (26,27). Our study was comprised of 28 (53.8\%) MRF positive, and 22 (42.3\%) EMVI positive patients. During the follow-up period, 11 patients $(21.1 \%)$ exhibited distant metastases, and the 2-year DFS was revealed to be $74.6 \%$, which is worse than the previous study on LARC (22).

This retrospective study has some limitations. Firstly, this was a single-center study with a limited patient number. Secondly, the pathological confirmation of LPLN was not available due to the absence of biopsies. The imaging diagnosis standard may have omitted some positive lymph nodes, while specific normal lymph nodes could have been over diagnosed, which potentially led to unnecessary treatment. Lastly, we used LRR and DFS as a measure of long-term outcomes due to the short follow-up duration even though DFS has been proven to be related to overall survival (28). A larger cohort and multicenter design are required for future studies with long term follow-up to acquire robust data.

In summary, the toxicities of LARC patients with clinically suspected positive LPLN treated by SIB-IMRT are acceptable. The absence of in-field lateral lymph node recurrence after NCRT combined with single TME, suggests that this treatment regime may be an optimal strategy for LARC patients with clinically suspected positive LPLN.

\section{Acknowledgments}

Funding: (I) Science Foundation of Peking University Cancer Hospital (No. 18-03); (II) Beijing Municipal Science \& Technology Commission (No. Z181100001718192); (III) Beijing Natural Science Foundation (No. 7182028); (IV) Beijing Municipal Administration of Hospitals' Youth Programme (No. QML20171104); (V) Sailing Project Clinical Technology Innovation Project (No. XMLX201842); (VI) Beijing Cancer Hospital project(A002053); (VII) Beijing Cancer Hospital project (2020-28).

\section{Footnote}

Reporting Checklist: The authors have completed the STROBE reporting checklist. Available at http://dx.doi. org/10.21037/atm-20-4040

Data Sharing Statement: Available at http://dx.doi. org/10.21037/atm-20-4040

Conflicts of Interest: All authors have completed the ICMJE uniform disclosure form (available at http://dx.doi. org/10.21037/atm-20-4040). The authors have no conflicts of interest to declare.

Ethical Statement: The authors are accountable for all aspects of the work in ensuring that questions related to the accuracy or integrity of any part of the work are appropriately investigated and resolved. Every patient signed an informed consent form before chemoradiotherapy. The study was conducted in accordance with the Declaration of Helsinki (as revised in 2013). The study was approved by the committee board of Beijing cancer Hospital (NO. 2020YJZ71).

Open Access Statement: This is an Open Access article distributed in accordance with the Creative Commons Attribution-NonCommercial-NoDerivs 4.0 International License (CC BY-NC-ND 4.0), which permits the noncommercial replication and distribution of the article with the strict proviso that no changes or edits are made and the original work is properly cited (including links to both the formal publication through the relevant DOI and the license). 
See: https://creativecommons.org/licenses/by-nc-nd/4.0/.

\section{References}

1. Chen $\mathrm{W}$, Zheng R, Baade PD et al. Cancer statistics in China, 2015. CA Cancer J Clin 2016;66:115-32.

2. Deng Y. Rectal Cancer in Asian vs. Western Countries: Why the Variation in Incidence? Curr Treat Options Oncol 2017;18:64.

3. Maas $M$, Nelemans PJ, Valentini $V$ et al. Long-term outcome in patients with a pathological complete response after chemoradiation for rectal cancer: a pooled analysis of individual patient data. Lancet Oncol 2010;11:835-44.

4. Capirci C, Valentini V, Cionini L et al. Prognostic value of pathologic complete response after neoadjuvant therapy in locally advanced rectal cancer: long-term analysis of 566 ypCR patients. Int J Radiat Oncol Biol Phys 2008;72:99-107.

5. Enker WE. Designing the optimal surgery for rectal carcinoma. Cancer 1996;78:1847-50.

6. Kaul S, Diamond GA. Good enough: a primer on the analysis and interpretation of noninferiority trials. Ann Intern Med 2006;145:62-9.

7. Pox C, Aretz S, Bischoff SC, et al. S3-guideline colorectal cancer version 1.0 Gastroenterol 2013;51:753-854.

8. Hida J, Yasutomi M, Fujimoto K et al. Does lateral lymph node dissection improve survival in rectal carcinoma? Examination of node metastases by the clearing method. J Am Coll Surg 1997;184:475-480.

9. Sauer I, Bacon HE. Influence of lateral spread of cancer of the rectum on radicability of operation and prognosis. Am J Surg 1951;81:111-20.

10. Sugihara K, Kobayashi H, Kato T et al. Indication and benefit of pelvic sidewall dissection for rectal cancer. Dis Colon Rectum 2006;49:1663-72.

11. Watanabe T, Itabashi M, Shimada Y et al. Japanese Society for Cancer of the Colon and Rectum (JSCCR) guidelines 2010 for the treatment of colorectal cancer. Int J Clin Oncol 2012;17:1-29.

12. Fujita S, Akasu T, Mizusawa J et al. Postoperative morbidity and mortality after mesorectal excision with and without lateral lymph node dissection for clinical stage II or stage III lower rectal cancer (JCOG0212): results from a multicentre, randomised controlled, non-inferiority trial. Lancet Oncol 2012;13:616-21.

13. Mori T, Takahashi K, Yasuno M. Radical resection with autonomic nerve preservation and lymph node dissection techniques in lower rectal cancer surgery and its results: the impact of lateral lymph node dissection. Langenbecks Arch Surg 1998;383:409-15.

14. Kusters M, Beets GL, van de Velde CJ et al. A comparison between the treatment of low rectal cancer in Japan and the Netherlands, focusing on the patterns of local recurrence. Ann Surg 2009;249:229-35.

15. Akiyoshi T, Matsueda K, Hiratsuka M, et al. Indications for Lateral Pelvic Lymph Node Dissection Based on Magnetic Resonance Imaging Before and After Preoperative Chemoradiotherapy in Patients with Advanced LowRectal Cancer. Ann Surg Oncol 2015;22 Suppl 3:S614-20.

16. Akiyoshi T, Ueno $M$, Matsueda $K$ et al. Selective lateral pelvic lymph node dissection in patients with advanced low rectal cancer treated with preoperative chemoradiotherapy based on pretreatment imaging. Ann Surg Oncol 2014;21:189-96.

17. Kim MJ, Kim TH, Kim DY et al. Can chemoradiation allow for omission of lateral pelvic node dissection for locally advanced rectal cancer? J Surg Oncol 2015;111:459-64.

18. Habr-Gama A, Sao Juliao GP, Vailati BB et al. Organ Preservation in cT2N0 Rectal Cancer After Neoadjuvant Chemoradiation Therapy: The Impact of Radiation Therapy Dose-escalation and Consolidation Chemotherapy. Ann Surg 2019;269:102-7.

19. Kim TG, Park W, Choi DH et al. Factors associated with lateral pelvic recurrence after curative resection following neoadjuvant chemoradiotherapy in rectal cancer patients. Int J Colorectal Dis 2014;29:193-200.

20. Lim SB, Yu CS, Kim CW et al. Clinical implication of additional selective lateral lymph node excision in patients with locally advanced rectal cancer who underwent preoperative chemoradiotherapy. Int J Colorectal Dis 2013;28:1667-74.

21. Suwinski R, Wzietek I, Tarnawski R et al. Moderately low alpha/beta ratio for rectal cancer may best explain the outcome of three fractionation schedules of preoperative radiotherapy. Int J Radiat Oncol Biol Phys 2007;69:793-9.

22. Li JL, Ji JF, Cai Y et al. Preoperative concomitant boost intensity-modulated radiotherapy with oral capecitabine in locally advanced mid-low rectal cancer: a phase II trial. Radiother Oncol 2012;102:4-9.

23. NCCN Clinical Practice Guidelines in Oncology, Colon Cancer (Version 3.2019). 2019 National Comprehensive Cancer Network, Inc. Accessed September 26, 2019. Available online: http://www.nccn.org 
24. Brown G, Richards CJ, Bourne MW et al. Morphologic predictors of lymph node status in rectal cancer with use of high-spatial-resolution MR imaging with histopathologic comparison. Radiology 2003;227:371-7.

25. Burbach JP, den Harder AM, Intven $M$ et al. Impact of radiotherapy boost on pathological complete response in patients with locally advanced rectal cancer: a systematic review and meta-analysis. Radiother Oncol 2014;113:1-9.

26. Nagtegaal ID, Quirke P. What is the role for the circumferential margin in the modern treatment of rectal

Cite this article as: Geng JH, Zhang YZ, Li YH, Li S, Wang L, Wang ZL, Zhu XG, Bu ZD, Li ZY, Su XQ, Cai Y, Wu AW, Wang WH. Preliminary results of simultaneous integrated boost intensity-modulated radiation therapy based neoadjuvant chemoradiotherapy on locally advanced rectal cancer with clinically suspected positive lateral pelvic lymph nodes. Ann Transl Med 2021;9(3):217. doi: 10.21037/atm-20-4040 cancer? J Clin Oncol 2008;26:303-12.

27. Chand M, Evans J, Swift RI et al. The prognostic significance of postchemoradiotherapy high-resolution MRI and histopathology detected extramural venous invasion in rectal cancer. Ann Surg 2015;261:473-9.

28. Valentini V, van Stiphout RG, Lammering G et al. Selection of appropriate end-points ( $\mathrm{pCR}$ vs $2 \mathrm{yDFS}$ ) for tailoring treatments with prediction models in locally advanced rectal cancer. Radiother Oncol 2015;114:302-9. 